\title{
In memoriam Peter Hans Dangel (1931-2021)
}

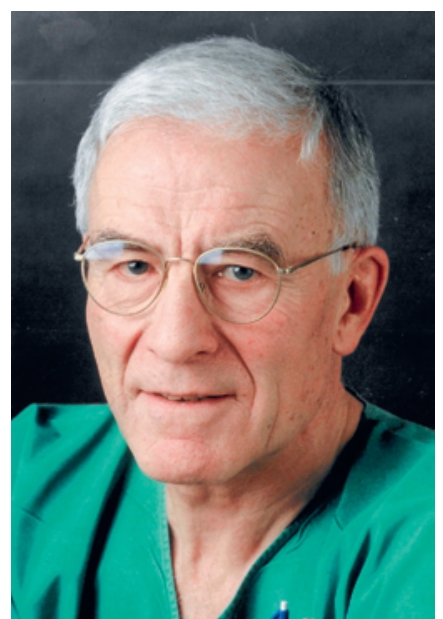

Dr. med. Peter H. Dangel ist am 14.12.2021 nach einem reich erfüllten Leben in seinem 90. Lebensjahr verstorben. Er war der Pionier der Kinderanästhesie und -intensivpflege in der Schweiz und hat die Entwicklung dieser medizinischen Fachgebiete initialisiert und wesentlich geprägt. Er studierte Medizin in Zürich und Paris. In seiner ersten Anstellung im Spital Samedan arbeitete er während zweier Jahre in Chirurgie, Innerer Medizin und Geburtshilfe. Anschliessend begann seine Ausbildung in Anästhesie bei Prof. Georg Hossli am Kantonsspital Zürich. Bereits nach sechs Monaten wurde er ins Kantonsspital Aarau versetzt als verantwortlicher Anästhesist! Danach war ein Jahr Innere Medizin im Spital Neumünster in Zürich vorgesehen, allerdings verbrachte er davon vier Monate im Kongo und arbeitete dort als Anästhesist. 1961 wurden ihm dann, nach einem viermonatigen Stage in der Anästhesieabteilung bei Prof. Martin Zindler in Düsseldorf der Aufbau und die Leitung einer Anästhesieabteilung im Kinderspital Zürich übertragen.

Bis zu diesem Zeitpunkt wurden Anästhesien in der Regel von Operationsschwestern, Medizinstudierenden oder chirurgischen Assistentinnen und Assistenten als Äthertropfnarkosen oder Chloroformnarkosen durchgeführt.

Im ersten Jahr führt er selbst oder zusammen mit Operationsschwestern 2500 Narkosen durch, darunter 20 Herzoperationen mit Herz-Lungen-Maschine oder Hypothermie. Innert kurzer Zeit erfolgten wesentliche Fortschritte bezüglich technischer Ausrüstung, Personal und verwendeter Substanzen. Narkosegeräte und Respiratoren wurden angeschafft. Cyclopropan und Halothan ersetzten Äther. Infusionstherapien wurden eingeführt via Venae sectio, später mit peripheren Kanülen und ab 1968 zum Teil mit zentralen Venenkathetern. Die kontinuierliche Überwachung vitaler Funktionen mit präkordialem Stethoskop und später mit EKG und maschineller Blutdruckmessung folgten. Die reine Inhalationsanästhesie wurde allmählich ersetzt und ergänzt durch Lachgas, Opiate, Muskelrelaxantien, Propofol und Nervenblockaden. Ketamin wurde für Notfalleingriffe und Verbrennungsbehand- lung benutzt. Die isovolämische Hämodilution hat er in den neunziger Jahren angewandt, um Bluttransfusionen zu vermeiden.

Parallel zur Entwicklung der Anästhesie wurde auch eine Intensivbehandlungsabteilung eingerichtet mit Langzeitbeatmung, totaler intravenöser Ernährung, Hirndruckmessung etc. Zusammen mit Prof. Gabriel Duc begann ab 1970 die spezifische intensivmedizinische Behandlung von Neu- und Frühgeborenen mit einer deutlichen Verbesserung der Prognose von $\mathrm{Pa}$ tientinnen und Patienten mit Atemnotsyndrom.

Ein weiterer Meilenstein war die enge Zusammenarbeit mit der Rega. Die Helikopterbasis kam 1971 auf das Dach des Kinderspitals, und sein ärztliches und pflegerisches Personal betreute die pädiatrischen Notfallpatientinnen und -patienten der Rega.

Peter Dangel war manuell in jeder Beziehung äusserst geschickt, und er lehrte seine Fähigkeiten und Kenntnisse unzähligen Assistenz- und Gastärztinnen und -ärzten. Seine wichtigste Aufgabe aber sah er in der Ausbildung des Pflegepersonals. Eine erste Anästhesieschwester bildete er 1961 aus, später folgten regelmässige Ausbildungskurse für Anästhesie- und Intensivpflegepersonal. Seine Schülerinnen bezeichneten sich auch später noch als «Dangelschwestern».

Er war Gründungsmitglied der Schweizerischen Gesellschaft für Intensivmedizin und hat 1995, nach dem Vorbild der Association of Paediatric Anaesthetists of Great Britain and Ireland, die Schweizerische Gesellschaft für Kinderanästhesie mitbegründet. Die Schweizerische Gesellschaft für Anästhesie und Reanimation hat ihn zum Ehrenmitglied ernannt.

Bis 1984 hat Peter Dangel die Intensivstation und bis zu seiner Pensionierung 1996 die Anästhesieabteilung geleitet. Er hat den Grundstein für eine Kinderanästhesieabteilung gelegt, welche unter seinen Nachfolgern internationale Grösse und Anerkennung erreicht hat Wir werden sein Andenken respektvoll bewahren und sprechen seiner Ehefrau und seiner Familie unser aufrichtiges Beileid aus.

KD Dr. med. Andreas Gerber Ehemaliger Chefarzt der Abteilung Kinderanästhesie Universitätskinderkliniken Zürich

Bildnachweis

E. Dangel 